\title{
Ab-initio Study of the Diffusion Coefficients in Fe-based Liquids
}

\author{
Miguel Fuentes-Cabrera ${ }^{1,2}$, Don Nicholson ${ }^{1}$, Mike Widom ${ }^{2}$, Yang Wang ${ }^{3}$ \\ and Marek Mihalkovic ${ }^{4}$ \\ ${ }^{1}$ Oak Ridge National Laboratory, P.O. Box 2008, Oak Ridge, TN, 37831 \\ ${ }^{2}$ Department of Physics, Carnegie Mellon University, PA 15213 \\ ${ }^{3}$ Pittsburgh Supercomputing Center, Carnegie Mellon University, Pittsburgh, PA 15213, \\ ${ }^{4}$ Institute of Physics, Slovak Academy of Science, Bratislava, Slovakia
}

\begin{abstract}
With atomistic force fields derived from ab-initio energies and atomic forces, we cooled $\mathrm{Fe}_{80} \mathrm{~B}_{20}$ from the liquid to the glass state. The pair-distribution functions and the diffusion coefficients were used to characterize the structural changes that $\mathrm{Fe}_{80} \mathrm{~B}_{20}$ underwent during the simulation. In the $\mathrm{FeFe}$ and $\mathrm{FeB}$ pair-distribution functions, when the temperature is lowered the first neighbor-peak becomes narrower and the secondneighbor peak splits at around 1000K. In the BB pair-distribution we observed that the first peak undergoes a significant change at the glass transition temperature, and that the first BB peak remains present at low temperatures. That the first BB peak exists at low temperature seems to contradict the prevailing view of the structure of transition metalmetalloid glasses.
\end{abstract}

\section{INTRODUCTION}

Despite the various and important applications that Bulk Metallic Glasses (BMG) could have, i.e. surgical blades, ships hulls, sporting goods, casing for consumers electronics, etc., there is not yet a clear understanding of the principles that guide their formation. Alloy designers use a set of empirical rules to select prospective BMG materials, but these rules fail often and the synthesis process is still inefficient. To solve this problem, we have designed a program directed toward understanding what guides glass formation. In this paper we demonstrate computational tools used to carry on systems-specific studies that can be directly compared to experiments.

$\mathrm{Fe}_{80} \mathrm{~B}_{20}$ does not form a bulk metallic glass but Poon et al. [1] has found that if $\mathrm{Zr}$ is added, the resulting $\mathrm{Fe}_{68} \mathrm{Zr}_{10} \mathrm{~B}_{22}$ does form a bulk glass. They suggested that the glass forming ability of $\mathrm{Fe}_{68} \mathrm{Zr}_{10} \mathrm{~B}_{22}$ is due to the presence of a $\mathrm{ZrB}$ backbone that enhances the stability of the undercooled melt. Our intention is to replicate Poon's experiment computationally. For this we have initiated the following plan: atomistic force fields will be developed to mimic ab-initio forces and energies in $\mathrm{Fe}_{80} \mathrm{~B}_{20}$ and $\mathrm{Fe}_{68} \mathrm{Zr}_{10} \mathrm{~B}_{22}$; both systems will be cooled from the liquid to the glass state and the glass transition temperature will be compared to experiments; during the simulations, the structural changes will be monitored to probe whether $\mathrm{Fe}_{68} \mathrm{Zr}_{10} \mathrm{~B}_{22}$ contains a $\mathrm{ZrB}$ backbone. In this paper we present preliminary results for $\mathrm{Fe}_{80} \mathrm{~B}_{20}$.

\section{METHODOLOGY AND RESULTS}

To carry on the simulations of $\mathrm{Fe}_{80} \mathrm{~B}_{20}$, we followed a three-step process: i) ab-initio data 
were obtained for Fe-B alloys, liquids and glasses with the Vienna Ab-initio Simulation Package (VASP) [2]; ii) the data were used to fit an atomistic force field of the Embedded Atom Method (EAM) type [3]; and iii) the EAM potential and the ParaDyn [4] code were used to cool $\mathrm{Fe}_{80} \mathrm{~B}_{20}$ from the liquid to the glass state. Next, these steps are described in more detail.

i) For $\mathrm{Fe}_{1-\mathrm{x}} \mathrm{B}_{\mathrm{x}}$ alloys, we studied stable, metastable and hypothetical structures, and obtained ab-initio enthalpies of formation, energy versus volume curves, and atomic forces. For $\mathrm{Fe}_{80} \mathrm{~B}_{20}$ liquids and glasses, we studied two liquids at $\mathrm{T}=1500 \mathrm{~K}$, one liquid at $\mathrm{T}=1400 \mathrm{~K}$, and two glasses [5], for which enthalpies of formation and atomic forces were obtained. The calculations were done with ultrasoft Vanderbilt type pseudopotentials as supplied by Kresse and Hafner [6], the exchange correlation functional of Perdew and Wang (1991), the Generalized Gradient Approximation, and the Vosko-Wilk-Nusair interpolation [7]. All calculations were performed with collinear spin-polarization. The local magnetic moments that are present in the liquid would be best modeled with a dynamic non collinear magnetic structure. This is beyond our current capability. Hence, we use a collinear treatment in order to include approximately local magnetic effects. Convergence studies were first carried out to optimize the plane wave energy cutoffs and the k-point grids to achieve $1 \mathrm{meV} / \mathrm{atom}$ accuracy in the total energy differences. We found that VASP's medium precision setting is enough while K-point grids of 8x8 8 up to $15 \times 15 \times 15$ were used for the crystalline structures. The liquid and glasses structures contain 100 atoms and the k-point set included only the $\Gamma$ point.

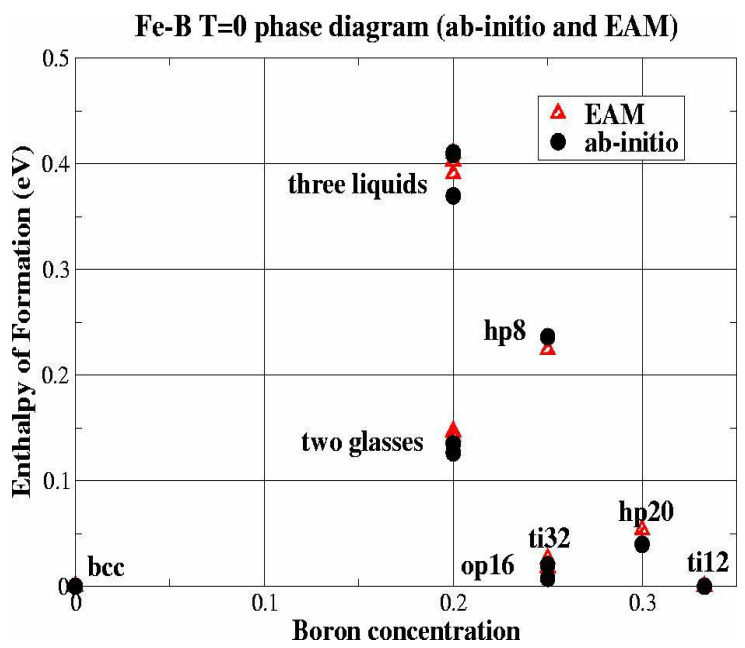

Figure 1 Ab-initio and EAM enthalpy of formation for $\mathrm{Fe}-\mathrm{B}$

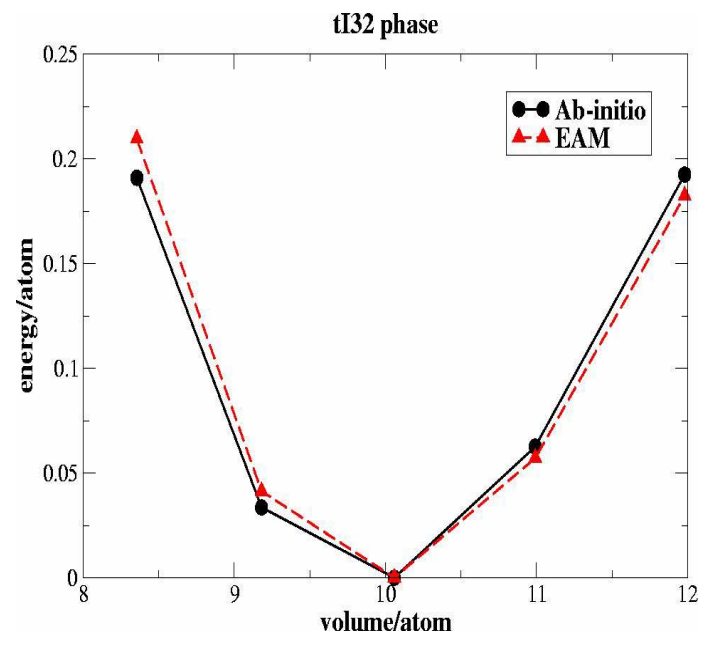

Figure $2 \mathrm{Ab}$ initio and EAM energy vs. volume curve for the tI32 structure 
ii) Within the EAM formulation, the energy of atom $i$ is given by:

$$
\begin{aligned}
& E_{i}=\frac{1}{2} \sum_{j \neq i} V_{i j}\left(r_{i j}\right)+F_{i}\left(n_{i}\right) \\
& n_{i}=\sum_{j \neq i} \rho_{j}\left(r_{i j}\right)
\end{aligned}
$$

$\mathrm{V}_{\mathrm{ij}}$ is the pair-potential between atoms $i$ and $j ; \mathrm{F}_{\mathrm{i}}$ and $n_{i}$ are the embedding energy and the "coordination-number" of atom $i$, respectively; $\rho_{\mathrm{j}}$ is the "atomic density" of atom $j$. In a binary system such as $\mathrm{FeB}$, there are three pair potentials, $\mathrm{V}_{\mathrm{FeFe}}, \mathrm{V}_{\mathrm{BB}}, \mathrm{V}_{\mathrm{FeB}}$, two atomic densities, $\rho_{\mathrm{Fe}}, \rho_{\mathrm{B}}$, and two embedding energies, $\mathrm{F}_{\mathrm{Fe}}, \mathrm{F}_{\mathrm{B}}$. We assumed that these 7 functions could be approximated by cubic splines, and their values were varied with a Monte Carlo technique until a good fit to the ab-initio data was obtained. That the EAM reproduces the ab-initio energies can be seen in Figs.1, 2 and Table 1. Figure 1 shows the $\mathrm{ab}$-initio and the EAM enthalpies of formation. Figure 2 shows the ab-initio and EAM energy versus volume curve for the structure tI32.

Table 1 contains a detail comparison between the ab-initio and EAM structural parameters of the crystalline structures. In Table 1 , the equilibrium volume $\mathrm{V}_{0}$, bulk modulus $\mathrm{B}_{0}$ and first derivative of the bulk modulus $\mathrm{B}_{0}$, were obtained by fitting the $\mathrm{ab}$ initio and EAM energy versus volume curves to the Birch-Murnaghan Equation of State [8].

\begin{tabular}{|l|l|l|c|}
\hline Structure type & \multicolumn{1}{|c|}{$\mathrm{V}_{0}$} & \multicolumn{1}{c|}{$\mathrm{B}_{0}$} & \multicolumn{1}{c|}{$\mathrm{B}_{0}$} \\
\hline BCC & $11.748(11.738)$ & $145.820(156.584)$ & $2.621(5.002)$ \\
\hline hP20 & $9.673(9.611)$ & $183.992(198.975)$ & $3.394(3.990)$ \\
\hline hP8 & $11.020(10.731)$ & $144.751(175.654)$ & $2.727(5.051)$ \\
\hline oP16 & $9.900(10.027)$ & $185.029(189.462)$ & $4.893(3.343)$ \\
\hline I12 & $9.297(9.196)$ & $208.336(208.783)$ & $1.182(4.024)$ \\
\hline tI32 & $9.941(9.879)$ & $194.926(195.125)$ & $4.325(4.261)$ \\
\hline
\end{tabular}

Table $1 A b$ initio and EAM structural parameters determined by a fitting to the Birch-Murnaghan Eauation of State

Table 2 contains the difference between the ab-initio and EAM atomic forces for the liquids. The ab-initio forces in relaxed crystal and glass structures are zero or extremely small. The EAM forces for the relaxed structures are zero in those cases where the force vanishes by symmetry and are small in other cases. The sizes of the forces in the liquid vary from atom to atom; the root mean square value is given in Table 2 . The difference between the ab-initio and EAM forces is significant but is of as the same order as that found by other researchers [9]. Furthermore, we have determined that the discrepancy is sufficiently small so as to preserve the dynamics as reflected by good agreement between diffusion calculated using EAM and ab-initio forces.

\begin{tabular}{|l|l|l|l|}
\hline Structure type & $\left.\left(<\left(\mathrm{F}_{\text {vasp }}\right)^{2}\right\rangle\right)^{1 / 2}$ & $\left.\left(<\left(\mathrm{F}_{\text {eam }}-\mathrm{F}_{\text {vasp }}\right)^{2}\right\rangle\right)^{1 / 2}$ & $\%$ Error \\
\hline $\mathrm{Fe}_{80} \mathrm{~B}_{20}$ liquid1 1500K & 1.971 & 0.478 & 24.25 \\
\hline $\mathrm{Fe}_{80} \mathrm{~B}_{20}$ liquid2 1500K & 2.140 & 0.567 & 26.5 \\
\hline $\mathrm{Fe}_{80} \mathrm{~B}_{20}$ liquid3 1400K & 2.022 & 0.572 & 28.29 \\
\hline
\end{tabular}

Table $2\left(<\left(\mathrm{F}_{\mathrm{vasp}}\right)^{2}>\right)^{1 / 2}$ is the root-mean square of the ab-initio force in eV/Angstrom. $\left(<\left(\mathrm{F}_{\text {eam }}-\mathrm{F}_{\text {vasp }}\right)^{2}>\right)^{1 / 2}$ is the root-mean square of the error in the force in eV/Angstrom. 
iii) The EAM potential and the ParaDyn code were used to cool $\mathrm{Fe}_{80} \mathrm{~B}_{20}$. Initially, the system was equilibrated at $1500 \mathrm{~K}$ by running a $3 \mathrm{~ns}$ simulation at constant pressure equal to zero. Then the temperature was lowered while keeping the pressure constant and equal to zero. The cooling rate used was, $10^{12} \mathrm{~K} / \mathrm{s}$ (every $100 \mathrm{ps}$ the temperature was lowered by $100 \mathrm{~K})$. Figure 3 shows the changes in the average volume with the temperature. The glass transition temperature is estimated to be around $900 \mathrm{~K}$ ( $750 \mathrm{~K}$ experimentally).

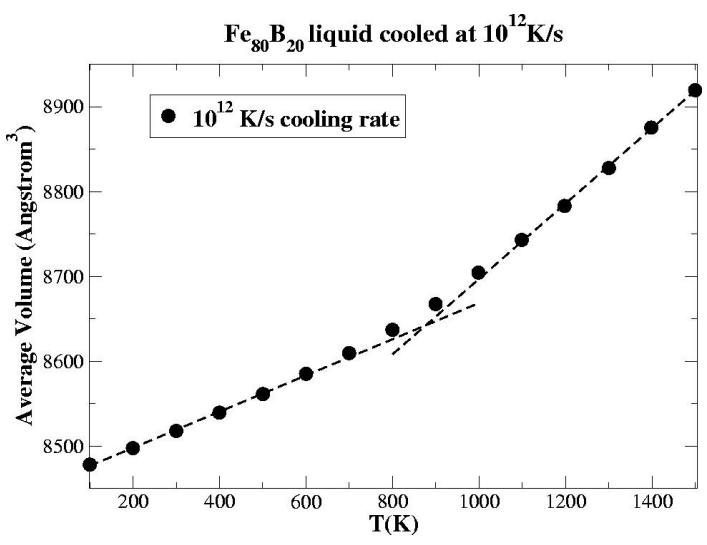

Figure 3 Cooling of 800 atoms of $\mathrm{Fe}_{80} \mathrm{~B}_{20}$ with EAM potentials and the ParaDyn code. The glass transition temperature is around $900 \mathrm{~K}$

\section{DISCUSSION}

Figures 4 and 5 show the $\mathrm{FeFe}$ and $\mathrm{FeB}$ pair-distribution functions calculated at different temperatures for a cooling rate of $10^{12} \mathrm{~K} / \mathrm{s}$. For clarity, the individual curves for different temperatures were displaced vertically. In both cases, it is seen that when the temperature is lowered the first neighbor-peak becomes narrower and the second neighbor-peak starts to split at $1000 \mathrm{~K}$.

Figure 6 shows the BB pair-distribution functions calculated at different temperatures for the cooling rate of $10^{12} \mathrm{~K} / \mathrm{s}$. If there were no chemical short range order, $20 \%$ of the $\mathrm{B}$ neighbors would on average be $\mathrm{B}$. We find that although chemical short range order reduces the number of B-B neighbors, a significant fraction $(6.7 \%$ at $1500 \mathrm{~K})$ of $\mathrm{B}$ neighbors are B. Also note that first-neighbor peak undergoes a sizable change at around $1000 \mathrm{~K}$. We suspect that this indicates large fluctuations in the B coordination near the glass transition temperature.

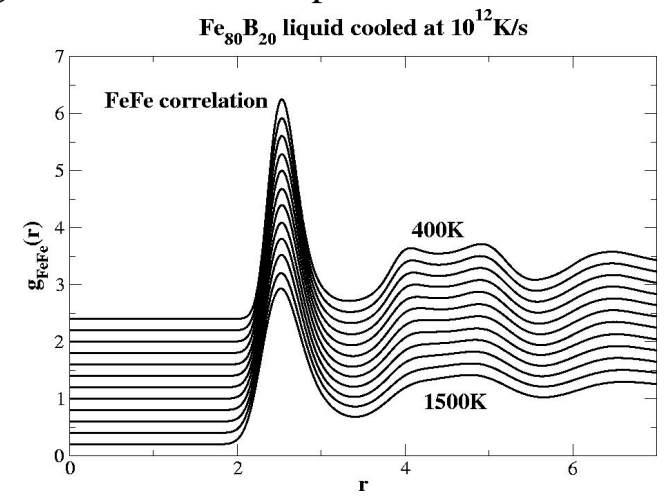

Figure $4 \mathrm{Fe}-\mathrm{Fe}$ partial pair distribution function.

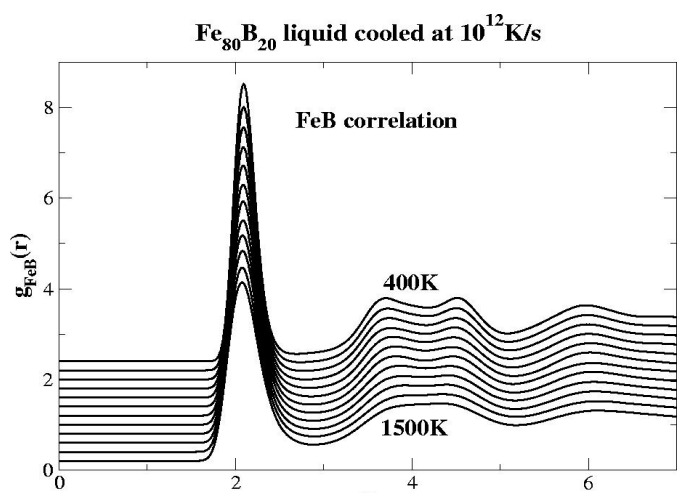

Figure 5 Fe-B partial pair distribution function. 


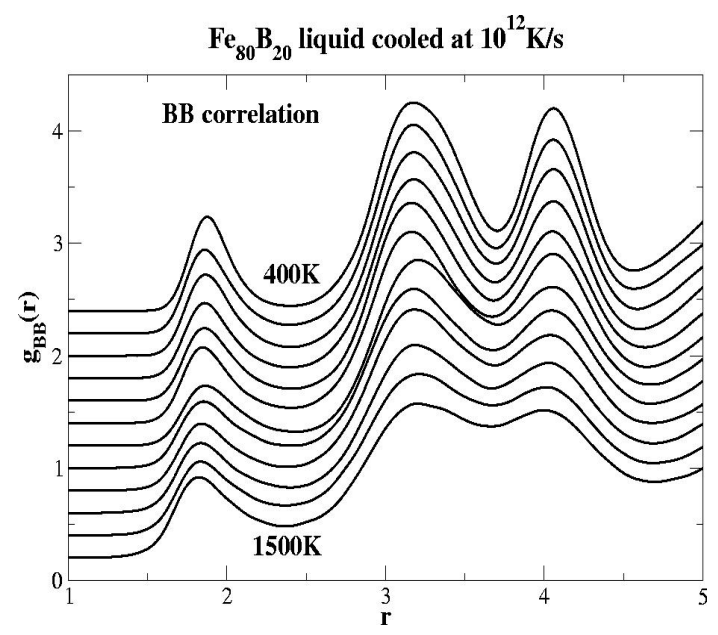

Figure 6 B-B partial pair distribution function,
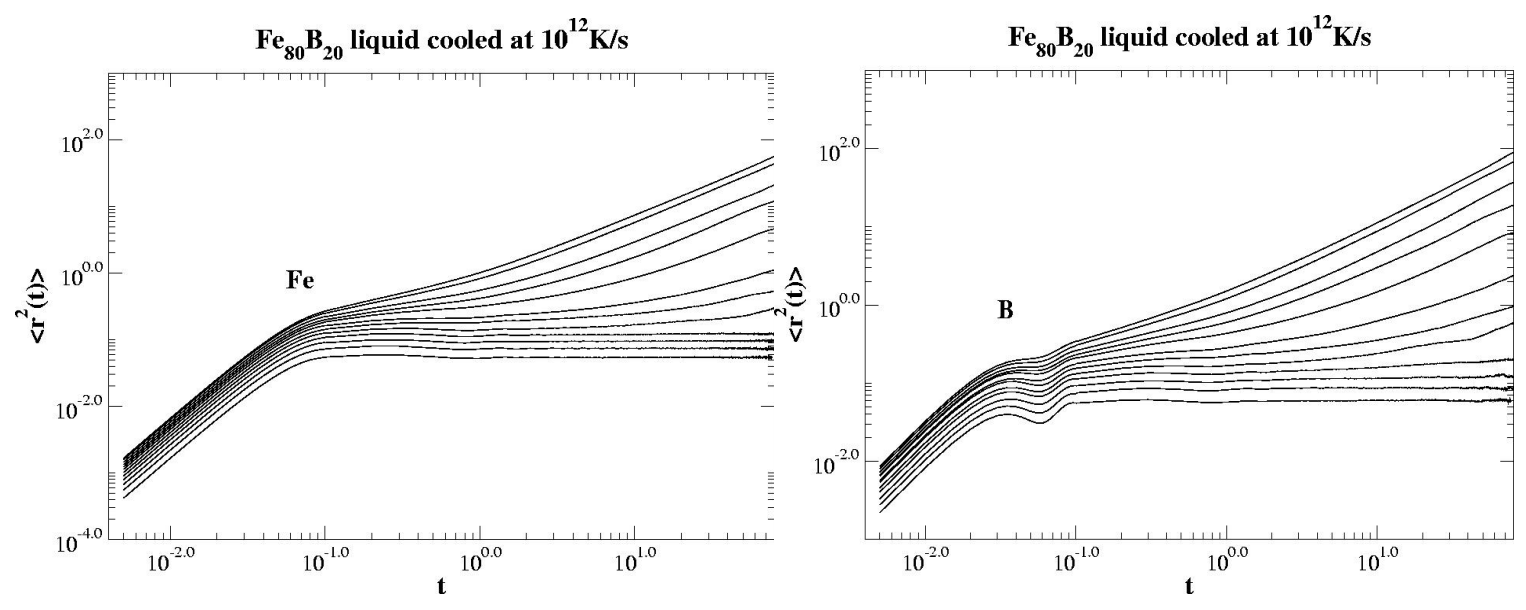

Figure $7 \mathrm{a}$ and $7 \mathrm{~b}$, root mean square displacement of $\mathrm{Fe}$ and $\mathrm{B}$ as a function of time for temperatures between $400 \mathrm{~K}$ (lower) and $1500 \mathrm{~K}$ (upper). Time is in ps.

Figures $7 \mathrm{a}$ and $7 \mathrm{~b}$ show the root-mean squared displacements versus time for $\mathrm{Fe}$ and $\mathrm{B}$ for all temperatures investigated. The upper(lower) curves correspond to $1500 \mathrm{~K}(400 \mathrm{~K})$. Consistent with B's smaller size and mass it is found to be a faster diffuser than Fe. The B curves have small minima near $0.05 p$ s which can be associated with B atoms that have escaped there local cage having a tendency to move back to the just vacated position. The diffusion drops rapidly near $1000 \mathrm{~K}$ where we observe the initiation of glass formation. On the time scale of our calculations the diffusion has ceased by $700 \mathrm{C}$.

\section{CONCLUSIONS}

We investigated the structural changes that bulk metallic glasses undergo upon cooling as reflected in the partial pair correlation functions. To this end, we created force-fields from ab-initio results and used these force-fields to cool multi-component systems from the liquid to the glass state. It is expected that future work of this type will help to select prospective bulk metallic glass materials efficiently. In this work we present results for $\mathrm{Fe}_{80} \mathrm{~B}_{20}$. With the EAM, we cooled $\mathrm{Fe}_{80} \mathrm{~B}_{20}$ and found that the first-neighbor BB peak i) 
undergoes a significant change at the glass transition temperature and ii) persists below the glass transition temperature. Analysis of B displacements suggested two regimes of B diffusion: above the glass transition temperature the $\mathrm{B}$ atoms diffuse with brief periods of contact with various other B whereas below the transition a subset of B moves in pairs. The fact that $\mathrm{BB}$ pairs exist below the glass transition temperature is in contradiction to the current view of the structure of transition metal-metalloid glasses [10], i.e. that in these types of glasses the average metalloid atom has no metalloid neighbor because it is enclosed within a Bernal hole [11] formed by Fe. This view that there are no B-B neighbors has been previously questioned based on a reexamination of experimental data [12]. The results presented here are a stepping stone for investigating the glass forming ability of $\mathrm{Fe}_{68} \mathrm{Zr}_{10} \mathrm{~B}_{22}$.

\section{ACKNOWLEDGEMENTS}

Work supported by DARPA/ONR Grant N00014-01-1-0961 under DOE subcontract DEAC05-00OR22R725464 with UT-Battelle, LLC.

\section{REFERENCES}

1. S.J. Poon, G.J. Shiflet, F.Q. Guo and V. Ponnambalam, J. of Non-Cryst. Solids 317, 1-9 (2003).

2. G. Kresse and J. Hafner, Phys. Rev. B 47, RC558 (1993); G. Kresse, PhD Thesis, Technische Universitat Wien 1993; G. Kresse and J. Furthmuller, Comput. Mat. Sci. 6 (1996) 15-50; G. Kresse and J. Furthmuller, Phys. Rev. B 54, 11169 (1996).

3. M.S. Daw and M.I. Baskes, Phys. Rev. B 29, 6443 (1984).

4. S.J. Plimpton and B.A. Hendrickson, Materials Theory and Modeling, Materials Research Society Proceedings, 291, 37, (1993).

5. The liquids and glasses structures studied were created with VASP. To create the liquids structures we first performed static relaxations in trial amorphous structures of $\mathrm{Fe}_{80} \mathrm{~B}_{20}$ and then increased the volume by $7 \%$-this value was chosen because it is slightly less than the reported volume ratio of $8 \%$ between the atomic volumes of $\mathrm{Fe}_{75} \mathrm{~B}_{25}$ liquid at $\mathrm{T}=1600 \mathrm{~K}$ and amorphous (Y. Waseda and H.S. Chen, Phys. Stat. Sol. A49, 387-392 (1978); N. Mattern, W. Matz and H. Hermann, Z. Nat. A43, 177-180 (1988).) Then we fixed the volume and carried on constant volume constant temperature molecular dynamics simulation at $\mathrm{T}=1500 \mathrm{~K}$ and $\mathrm{T}=1400 \mathrm{~K}$. From all the liquids structures generated, we selected two configurations at $\mathrm{T}=1500 \mathrm{~K}$ and one configuration at $\mathrm{T}=1400 \mathrm{~K}$. To create the glasses structures, we set the temperature equal to zero and relaxed the structure. From all the glasses structures generated we selected only two configurations.

6. D. Vanderbilt, Phys. Rev. B 41, 7892 (1990); G. Kresse and J. Hafner, J. Phys.: Condens. Matter 6, 8245 (1992).

7. S.H. Vosko, L. Wilk, and M. Nusair, Can. J. Phys. 58, 1200 (1980).

8. Birch F. J. Geophys. Res. 47, 227 (1952).

9. Xiang-Yang Lui, James B. Adams, Furio Ercolessi, and John A. Moriarty, Modeling Simul. Mater. Sci. Eng. 4293 (1996).

10. E. Nold, P. Lamparter, H. Olbrich, g. Rainer-Harbach, and S. Steeb, Z. Naturforsch 36a 1032 (1981).

11. J. D. Bernal, Nature 185, 68 (1960).

12. N. Cowlam, J. Non. Cryst. Sol. 207II 567 (1996). 